\title{
Immune-inflammatory markers and arterial stiffness indexes in subjects with acute ischemic stroke with and without metabolic syndrome
}

\author{
Antonino Tuttolomondo ${ }^{1 *}$, Rosaria Pecoraro ${ }^{1}$, Domenico Di Raimondo ${ }^{1}$, Riccardo Di Sciacca ${ }^{1}$, Baldassare Canino ${ }^{1}$, \\ Valentina Arnao ${ }^{2}$, Carmelo Buttà ${ }^{1}$ Vittoriano Della Corte ${ }^{1}$, Carlo Maida', Giuseppe Licata ${ }^{1}$ and Antonio Pinto ${ }^{1}$
}

\begin{abstract}
Objective: The aim of our study was to evaluate the associations between arterial stiffness indexes and immune-inflammatory markers in subjects with acute ischemic stroke with and without metabolic syndrome.

Materials/Methods: We enrolled 130 patients with acute ischemic stroke and metabolic syndrome, 127 patients with acute ischemic stroke without metabolic syndrome and 120 control subjects without acute stroke. Applanation tonometry was used to record the augmentation index (Aix) and pulse wave velocity (PWV). We also evaluated plasma levels of C-reactive protein (CRP), Interleukin-1 beta (IL-1ß), tumor necrosis factor-alpha (TNF-a), Interleukin-6 (IL-6) and Interleukin-10 (IL-10), E-selectin, P-selectin, intercellular adhesion molecule-1 (ICAM-1), vascular cell adhesion molecule-1 (VCAM-1), von Willebrand Factor (VWF) plasma levels, tissue plasminogen activator (TPA) and plasminogen activator inhibitor-1 (PAl-1).

Results: In subjects with acute ischemic stroke and metabolic syndrome we observed higher median plasma values of immuno-inflammatory markers. In acute ischemic stroke patients and metabolic syndrome in relation of each TOAST subtype we observed a more significant positive correlation between PWV and immuno-inflammatory markers.

Conclusions: Stroke subjects with acute ischemic stroke and metabolic syndrome showed a higher degree of immuno-inflammatory and arterial stiffness indexes possibly due to metabolic background of these types of patients that trigger a more intense immune-inflammatory activation irrespective of stroke subtype, whereas being related to stroke subtype in subjects without metabolic syndrome.
\end{abstract}

Keywords: Stroke, Arterial stiffness, PWW, Aix, Cytokines

\section{Introduction}

Arterial stiffness is increasingly recognized as an important determinant of cardiovascular risk [1,2] and may be directly involved in the process of atherosclerosis [3]. Factors underlying increased arterial stiffness are not completely understood, but both functional and structural alterations in the vessel wall are thought to be important. Acute and chronic inflammation are also associated with endothelial dysfunction [4,5]. More recently, inflammation has been associated with an increased risk

\footnotetext{
* Correspondence: bruno.tuttolomondo@unipa.it

${ }^{1}$ Dipartimento Biomedico di Medicina Interna e Specialistica, Università degli Studi di Palermo, P.zza delle Cliniche n.2, 90127 Palermo, Italy

Full list of author information is available at the end of the article
}

of cardiovascular events and the level of C-reactive protein (CRP), a marker of inflammation, predicts outcome in patients with cardiovascular disease [6,7]. Taken together, these data suggest that inflammation may be a key cause of cardiovascular damage.

Although there are some inconsistencies, a number of recent studies have suggested that in a healthy population, there may be a significant relationship between hs-CRP and measures of arterial stiffness [8,9]. Yasmin et al. found hs-CRP to be related to pulse wave velocity (PWV) but not to Augmentation Index (AIx) [10]. In contrast, Kampus et al. found hs-CRP to be independently and significantly associated with AIx but not with the timing of the reflected pressure wave (RT) [8]. However, in 
inflammatory conditions, the relationship may be stronger. In patients with systemic vasculitis, in which hsCRP levels are markedly elevated, they were positively correlated with PWV and AIx [9].

Metabolic syndrome is a cluster of common pathologies: abdominal obesity linked to an excess of visceral fat, insulin resistance, dyslipidemia and hypertension [11]. At the molecular level, metabolic syndrome is accompanied by dysregulation in the expression of adipokines (cytokines and chemokines) making metabolic syndrome an inflammatory condition. The molecular mechanism underlying the relationship between metabolic syndrome and neurological disorders is not fully understood. However, it is becoming increasingly evident that all cellular and biochemical alterations observed in metabolic syndrome such as impairment of endothelial cell function, abnormality in essential fatty acid metabolism and alterations in lipid mediators along with abnormal insulin/leptin signaling may represent a pathological bridge between metabolic syndrome and neurological disorders such as stroke.

The association of MetS with arterial stiffness has been investigated in many studies [12-14]; however, most of these studies were cross-sectional [14].

In a recent study [15] we reported that stroke patients with metabolic syndrome, compared to stroke subjects without metabolic syndrome, and compared to control subjects without stroke showed a higher mean PWV. We also showed in the same study that PWV value in lacunar subjects with metabolic syndrome was significantly higher compared to value observed in subjects with lacunar stroke without metabolic syndrome. In the same study we reported that PWV in subjects with atherosclerotic and cardioembolic subtypes of stroke and metabolic syndrome was significantly higher compared to values observed in subjects with these stroke subtypes and without metabolic syndrome.

Thus it's presumable that these findings observed in stroke patients with metabolic syndrome could be due to inflammatory and metabolic background of subjects with metabolic subjects that could influence acute inflammatory activation after an acute ischemic stroke and arterial stiffness process.

On this basis, as an extension of this previous study and on the same sample of patients, we analyzed plasma levels of some immuno-inflammatory markers with the aim to evaluate the relationships between arterial stiffness and immune-inflammatory markers such as cytokines, selectins and adhesion molecules in subjects with acute ischemic stroke with and without metabolic syndrome.

\section{Materials and methods Patient selection}

Patients were considered as having metabolic syndrome if they had $\geq 3$ of the following components, based on modification of the NCEP/ATP III: hypertension defined as systolic blood pressure $\geq 130 \mathrm{~mm} / \mathrm{Hg}$ and/or a diastolic blood pressure $\geq 85 \mathrm{~mm} / \mathrm{Hg}$, and by history of hypertension, raised plasma triglycerides $(\geq 150 \mathrm{mg} / \mathrm{dL}$ [1.7 mmol/L]), low high-density lipoprotein cholesterol levels ( $\geq 40 \mathrm{mg} / \mathrm{dL}$ in men and $\geq 50 \mathrm{mg} / \mathrm{dL}$ in women), and impaired fasting glucose defined as fasting plasma glucose concentration $\geq 110 \mathrm{mg} / \mathrm{dL}(6.1 \mathrm{mmol} / \mathrm{L})$, but less than the criteria for diabetes of $\geq 125 \mathrm{mg} / \mathrm{dL}$ $(\geq 7.0 \mathrm{mmol} / \mathrm{L})$. As a definition of obesity, we used a body mass index $\geq 30 \mathrm{~kg} / \mathrm{m} 2$ instead of WHR on a basis of modification of the NCEP/ATP III [16].

We enrolled all consecutive patients with a diagnosis of acute ischemic stroke admitted to the Internal Medicine Department at the University of Palermo between November 2009 and January 2011, and, as controls, hospitalized patients with acute ischemic stroke without a diagnosis of metabolic syndrome and hospitalized patients without a diagnosis of acute ischemic stroke, admitted, in the same period, to our Internal Medicine Department for any cause other than acute cardiovascular and cerebrovascular events.

Stroke was defined by focal neurological signs or symptoms thought to be of vascular origin that persisted for $>24$ hours, confirmed by brain CT and/or MRI in baseline conditions and brain $\mathrm{CT}$ with contrast medium after 48-72 hours [17].

In order to match patients with acute ischemic stroke and controls for cardiovascular risk and previous cardiovascular morbidity, controls were included if they had vascular risk factors or a history of myocardial infarction or cerebro-vascular disease or peripheral vascular disease, but they were excluded if they had either current or recent (within six months) cerebro-vascular disease or one of the exclusion criteria (see above).

Cardiovascular risk factors were evaluated for both cases and controls on the basis of the criteria shown below. Hypercholesterolemia was defined as the presence of total cholesterol blood levels $\geq 200 \mathrm{mg} / \mathrm{dL}$. Hypertension was defined as present if subjects had been previously diagnosed according to the World Health Organization/International Society of Hypertension guidelines and were routinely receiving antihypertensive therapy.

Patients were defined as type 2 diabetics if they had known diabetes treated by diet, oral hypoglycemic drugs or insulin before stroke.

Previous coronary artery disease was determined on the basis of a history of physician-diagnosed angina, myocardial infarction, or any previous revascularization procedure assessed by a questionnaire.

Previous cerebro-vascular disease (TIA/ischemic stroke) was assessed by history, specific neurologic examination performed by specialists, and hospital or radiological (brain 
computer tomography or brain magnetic resonance) records of definite previous stroke.

Subjects were classified as having previous peripheral artery disease (PAD) when they had a history of ABI $<0.9$ and/or of intermittens claudicatio or of critical limb ischemia or when they had undergone a peripheral arterial bypass or amputation.

The study protocol was approved by the Policlinico Universitario "P. Giaccone" Ethical Committee of Palermo, and all participants gave written informed consent. Every subject with ischemic stroke was matched for age ( \pm 3 years), sex, and cardiovascular risk factor prevalence with one control subject.

The type of acute ischemic stroke was classified according to the TOAST classification [18]: 1) Large Artery AtheroSclerosis (LAAS); 2) CardioEmbolic Infarct (CEI); 3) LACunar infarct (LAC); 4) stroke of Other Determined Etiology (ODE); 5) stroke of UnDetermined Etiology (UDE).

All the ischemic stroke patients underwent: medical history with recording of potential stroke risk factors, blood and coagulation tests, 12-lead ECG, 24 hour electrocardiography monitoring, trans-thoracic echocardiography, carotid ultrasound, brain CT or MRI at admission (repeated between the third and seventh days of stroke onset).

Neurological deficit score on admission was evaluated by Scandinavian Stroke Scale (SSS). SSS assesses neurological deficit through an evaluation of consciousness level, eye movement, strength in arms, hands, and legs, orientation, language, facial weakness and gait, giving rise to a score ranging from 58 (absence of deficit) to 0 (death).

\section{PWV measurement}

Carotid-femoral PWV was measured in the supine position using the automatic device (SphygmoCor version 7.1) that measured the time delay between the rapid upstroke of the carotid and femoral artery pulse waves. The distance between the 2 arterial points was measured on the surface of the body using a tape measure. PWV was calculated as the distance traveled by the arterial pulse wave (meters) divided by the time delay between the 2 arterial points (seconds), thus expressed as meters per second. The "distance between the 2 arterial points" was measured using the total distance between the carotid and femoral sites of measurement [19].

\section{Pulse Wave analysis}

Applanation tonometry was used to record radial artery pressure waveform continuously, and mean values of $\geq 2$ screens of pulse waves of good quality were used for analysis. On the basis of the collected data, an averaged radial pressure waveform was generated and a corresponding aortic pressure waveform and BP calculated by the validated transfer function (SphygmoCor version 7.1). The aortic pressure waveform was used to calculate the AIx (difference in height between the first and second systolic peaks expressed as a percentage of pulse pressure (PP).

\section{Laboratory evaluation}

Blood samples were obtained in the non-fasting state. After 10 minutes of rest in the supine position, vital signs were recorded and blood samples were collected from the antecubital vein.

EDTA-anticoagulated peripheral blood was drawn from each patient within 12 hours from symptom onset. Serum and plasma were immediately separated by centrifugation and stored in aliquots at $-80^{\circ} \mathrm{C}$ until analysis.

We evaluated plasma levels of C-reactive protein (CRP), Interleukin-1beta (IL-1 $\beta$ ), tumor necrosis factoralpha (TNF- $\alpha$ ), Interleukin-6 (IL-6) and Interleukin-10 (IL-10), E-selectin, P-selectin, intercellular adhesion molecule-1 (ICAM-1), vascular cell adhesion molecule-1 (VCAM-1), as markers of immune-inflammatory activation, von Willebrand Factor (vWF) plasma levels as a marker of endothelial dysfunction, tissue plasminogen activator (TPA) and plasminogen activator inhibitor-1 (PAI-1) as marker of thrombotic/ fibrinolytic pathway.

IL-1 $\beta$, TNF- $\alpha$, IL- 6 and IL-10 and VWF antigen were measured using a sandwich ELISA (Human IL-1 $\beta$, TNF$\alpha$, IL-6 and IL-10 Quantikine, R\&D Systems (VWF ELISA kitdurian, Instrumentation Laboratory, Milano, Italy); VCAM-1, ICAM-1, E-selectin, P-selectin, PAI-1 and TPA-antigen were measured by commercial bioimmunoassay (Human sICAM-1, sVCAM-I, sE-selectin and sP-selectin Parameter, Quantikine, R\&D Systems, Gentaur AssayMax Human Plasminogen Activator Inhibitor-1 (PAI-1) ELISA Kit, Gentaur AssayMax Tissue Plasminogen Activator (TPA) ELISA Kit).

The minimum detectable concentrations for the diagnostic tests are: TNF alpha: $1.6 \mathrm{pg} / \mathrm{mL}$; IL-1 $\beta$ : $<1 \mathrm{pg} /$ $\mathrm{mL}$; IL-6: <0.70 pg/mL; IL-10: >3.9 pg/mL; ICAM-1: < $0.35 \mathrm{ng} / \mathrm{mL}$; VCAM-1: $0.6 \mathrm{ng} / \mathrm{mL}$; E-Selectin: $<0.1 \mathrm{ng} /$ mL; P-Selectin: < $0.5 \mathrm{ng} / \mathrm{mL}$; vWF: $1.0 \%$; TPA: $0.3 \mathrm{pg} /$ $\mathrm{ml}$; PAI- $1:<50 \mathrm{pg} / \mathrm{ml}$.

Intraassay and interassay coefficients of variation were: TNF alpha: $4.2 \%$ and 4.6\%; IL-1ß: $3.3 \%$ and $4.2 \%$; IL-6: 1.6\% and 3.3\%; IL-10: 4.3\% and 7.5\%; ICAM-1: $4.8 \%$ and 6.1\%; VCAM-1: $3.5 \%$ and $7.7 \%$; E-Selectin: $4.8 \%$ and 5.7\%; P-Selectin: $4.9 \%$ and $8.8 \%$; vWF: $5 \%$ and $10 \%$; TPA: $4.8 \%$ and $5 \%$; PAI- $1: 5.7 \%$ and $8.3 \%$.

\section{Statistical analysis}

Results are expressed as mean \pm SD for continuous variables and percentages for categorical data, with $P \geq 0.05$ considered significant. 
Analysis of normality was performed with the ShapiroWilk W test.

Non-normally distributed data were logarithmically (Log10) transformed before analysis.

The relationship between immune-inflammatory markers, PWV, AIx, and other parameters was analyzed using nonparametric methods (Spearman p correlations) after correction for age and gender.

\section{Results}

We enrolled 130 patients with acute ischemic stroke and metabolic syndrome, 127 patients with acute ischemic stroke without metabolic syndrome and 120 control subjects matched for age, sex, cardiovascular risk factors and previous cardiovascular morbidity.

In patients with acute ischemic stroke and metabolic syndrome according to the TOAST criteria, the etiology of stroke was: large-artery atherosclerosis (LAAS) in 49 (37.69\%) patients, cardioembolism (CEI) in 45 (34.61\%) patients, lacunar stroke in $32(24.61 \%)$ patients whereas $3(2.3 \%)$ subjects were classified as ODE and 2 UDE $(0.76 \%)$

In patients with acute ischemic stroke without metabolic syndrome, according to the TOAST criteria, the

Table 1 Premorbid cardiovascular risk factors, clinical characteristics and medication

\begin{tabular}{|c|c|c|c|c|}
\hline Variable & $\begin{array}{l}\text { Stroke pts with metabolic } \\
\text { syndrome (n: 130) }\end{array}$ & $\begin{array}{l}\text { Stroke pts without metabolic } \\
\text { syndrome (n: 127) }\end{array}$ & Controls (n: 109) & $P$ \\
\hline Age (years) & $64(61-72.5)$ & $72(69-83.5)$ & $69(63-80)$ & 0.031 \\
\hline$M / F(n)$ & $69 / 61$ & $60 / 67$ & $54 / 55$ & 0.027 \\
\hline $\mathrm{SBP} / \mathrm{DBP}(\mathrm{mm} / \mathrm{Hg})$ & $152 \pm 7.9 / 98 \pm 6.2$ & $147 \pm 8.9 / 92 \pm 3.2$ & $141 \pm 9.8 / 91 \pm 2.2$ & $<0.001$ \\
\hline diabetes (n/\%) & $63(48.46)$ & $39(30 \%)$ & $47(41.28 \%)$ & 0.263 \\
\hline Hypertension (n/\%) & 66 (50.79\%) & $47(37 \%)$ & $48(44.03 \%)$ & 0.301 \\
\hline Glucose blood levels (mg/dl) & $149.3(97-186)$ & $129.1(88-142)$ & $102(81.5-133.5)$ & $<0.001$ \\
\hline Cholesterol blood levels (mg/dl) & $241(189-270)$ & $222(179-245)$ & $210(167-225)$ & $<0.001$ \\
\hline Triglyceride blood levels (mg/dl) & $197.5(149.75-210.75)$ & $157.5(119.4-181.75)$ & $167.5(139.4-192.1)$ & 0.004 \\
\hline White blood cells (per $\mathrm{mm}^{3}$ ) & $9100(6200-11000)$ & $8200(7100-10500$ & $7400(6500-9800)$ & $<0.001$ \\
\hline \multicolumn{5}{|l|}{ Stroke subtype } \\
\hline$\cdot$ LAAS & $49(37.69)$ & $52(40.94)$ & & \\
\hline - Lacunar & $45(34.61)$ & $37(29.7)$ & & \\
\hline • CEI & $32(24.61)$ & $35(27.55)$ & & \\
\hline$\cdot$ ODE & $3(2.3)$ & $2(1.57)$ & & \\
\hline - UDE & $1(0.76)$ & $1(0.78)$ & & \\
\hline Alx (\%) & $105 \pm 3.5$ & $101 \pm 4.1$ & $89 \pm 4.6$ & $<0.001$ \\
\hline $\mathrm{PWV}(\mathrm{m} / \mathrm{s})$ & $12.9 \pm 3.3$ & $11.2 \pm 3.2$ & $10.02 \pm 2.29$ & $<0.001$ \\
\hline SSS & $27.12 \pm 16.21$ & $29.65 \pm 19$ & $30 \pm 16.21$ & \\
\hline IL-1-beta (pg/ml) & $10(6-11)$ & $8(5-16)$ & $4(2-5)$ & $<0.001$ \\
\hline IL-6 (pg/ml) & $12(9-31)$ & $10.3(7-29)$ & $8(3.1-12)$ & $<0.001$ \\
\hline TNF-a (pg/ml) & $35.5(10.25-46)$ & $26(10.55-49)$ & $5.1(1.1-4.3)$ & $<0.001$ \\
\hline E-selectin (ng/ml) & $4.05(2.0-5.8)$ & $2.1(1.0-3.8)$ & $2(1-2)$ & $<0.001$ \\
\hline P-selectin (ng/ml) & $6.5(3-8.9)$ & $4.5(2-6.8)$ & $3,1(2.1-4)$ & 0.004 \\
\hline VICAM-1 (ng/ml) & $18(12.1-23)$ & $16(10.1-20)$ & $10(7-15)$ & $<0.001$ \\
\hline ICAM-1 (ng/ml) & $17.8(12.2-20)$ & $13.5(12.2-20)$ & $10.9(12-16.1)$ & $<0.001$ \\
\hline IL-10 (pg/ml) & $2.05(2-7)$ & $3.15(2-7)$ & $5(2-10)$ & 0.031 \\
\hline vWF (ng/ml) & $11(6-15)$ & $4(3-9)$ & $2(1-5)$ & 0.0001 \\
\hline PAl-1 (pg/ml) & $137(99.5-155)$ & $23(11-24)$ & $15(7-22)$ & $<0.001$ \\
\hline TPA (pg/ml) & $21(10.55-39)$ & $55(29-88)$ & $11(8-19)$ & 0.44 \\
\hline CHF (n/\%) & $23(17.69)$ & $20(15.74)$ & $15(14.70)$ & 0.065 \\
\hline
\end{tabular}

SBP: systolic blood pressure; DBP: diastolic blood pressure; MAP: mean arterial pressure; Alx: augmentation index; PWV: pulse wave velocity; SSS: Scandinavian Stroke Scale score; NIHSS: National Institutes of Health Stroke Scale; Angiotensin II receptor blockers (ARBs); CAD: coronary artery disease; CHF: congestive heart failure; TIA: transitory ischemic attack; LVH: left ventricular hypertrophy; WMHLS: white matter hypertension lesions.

Demographic and history data are expressed as $\mathrm{n}^{\circ}$ (percentage). 
etiology of stroke was large-artery atherosclerosis (LAAS) in 52 (40.94\%) patients, cardioembolism (CEI) in 37 (29.7\%) patients, lacunar stroke in 35 (27.55\%) patients whereas $1(1.57 \%)$ subjects were classified as ODE and 1 $(0.78 \%)$ as UDE.

Baseline characteristics in stroke patients and in relation to each TOAST subtype are given in Tables 1 and 2 .

In subjects with acute ischemic stroke and metabolic syndrome compared to subjects with acute ischemic stroke without metabolic syndrome and control subjects we observed higher median plasma values of IL-1-beta $[10(6-11) \mathrm{pg} / \mathrm{ml})$ vs. $8(5-16) \mathrm{pg} / \mathrm{ml}$ vs. $4(2-5) \mathrm{pg} / \mathrm{ml}$; $\mathrm{p}<0.001]$; IL-6 [12 (9-31) pg/ml) vs. $10.3(7-29) \mathrm{pg} / \mathrm{ml}$ vs. $8(3.1-12) \mathrm{pg} / \mathrm{ml} ; \mathrm{p}<0.001]$; TNF- $\alpha 35.5(10.25-46)$ $\mathrm{pg} / \mathrm{ml})$ vs. $26(10.55-49) \mathrm{pg} / \mathrm{ml}$ vs. $5.1(1.1-4.3) \mathrm{pg} / \mathrm{ml}$; $\mathrm{p}<0.001]$; E-selectin [4.05 (2.0-5.8) ng/ml) vs. 2.1 (1.0$3.8) \mathrm{ng} / \mathrm{ml}$ vs. $2(1,2) \mathrm{ng} / \mathrm{ml} ; \mathrm{p}<0.001]$; P-selectin $[6.5$ $(3-8.9) \mathrm{ng} / \mathrm{ml})$ vs. $4.5(2-6.8) \mathrm{ng} / \mathrm{ml}$ vs. $3,1(2.1-4) \mathrm{ng} / \mathrm{ml}$; $\mathrm{p}<0.001]$; VICAM-1 [18 (12.1-23) $\mathrm{ng} / \mathrm{ml})$ vs. 16 (10.120) $\mathrm{ng} / \mathrm{ml}$ vs. 10 (7-15) ng/ml; $\mathrm{p}<0.001]$; ICAM-1 [17.8 $(12.2-20) \mathrm{ng} / \mathrm{ml})$ vs. $13.5(12.2-20) \mathrm{ng} / \mathrm{ml}$ vs. 10.9 (1216.1) $\mathrm{ng} / \mathrm{ml} ; \mathrm{p}<0.001]$; vWF [17.8 (12.2-20) $\mathrm{ng} / \mathrm{ml}) \mathrm{vs.}$ $13.5(12.2-20) \mathrm{ng} / \mathrm{ml}$ vs. $10.9(12-16.1) \mathrm{ng} / \mathrm{ml} ; \mathrm{p}<0.001]$; PAI-1 [137 (99.5-155) vs. $23(11-24) \mathrm{ng} / \mathrm{ml}$ vs. 10.915

Table 2 Laboratory and clinical variables and arterial stiffness markers by ischemic stroke subtype in subjects with ischemic stroke and metabolic syndrome and in patients with acute ischemic stroke without metabolic syndrome

\begin{tabular}{|c|c|c|c|c|c|c|c|c|c|}
\hline \multirow{2}{*}{$\begin{array}{l}\text { Variable } \\
\text { Number }\end{array}$} & \multicolumn{2}{|c|}{ Lacunar } & \multirow[t]{2}{*}{$p$} & \multicolumn{2}{|c|}{ LAAS } & \multirow[t]{2}{*}{$p$} & \multicolumn{2}{|c|}{ CEI } & \multirow[t]{2}{*}{$p$} \\
\hline & $\begin{array}{c}\text { With } \\
\text { metabolic } \\
\text { syndrome } \\
\text { (n: } 49)\end{array}$ & $\begin{array}{c}\text { Without } \\
\text { metabolic } \\
\text { syndrome } \\
\text { (n 52) }\end{array}$ & & $\begin{array}{c}\text { With } \\
\text { metabolic } \\
\text { syndrome } \\
\text { (n: 45) }\end{array}$ & $\begin{array}{l}\text { Without } \\
\text { metabolic } \\
\text { syndrome } \\
\text { (n: } 37)\end{array}$ & & $\begin{array}{c}\text { With } \\
\text { metabolic } \\
\text { syndrome } \\
(\mathrm{n}: 32)\end{array}$ & $\begin{array}{c}\text { Without } \\
\text { metabolic } \\
\text { syndrome } \\
\text { (n:35) }\end{array}$ & \\
\hline SBP/DBP (mm/Hg) & $148 \pm 6.5 / 94 \pm 4.5$ & $144 \pm 45 / 9235$ & 0.020 & $146 \pm 3.4 / 93 \pm 4.5$ & $145.5 / 91 \pm 2.9$ & 0.89 & $144 \pm 3.8 / 90 \pm 3.1$ & $143 \pm 2.8 / 90 \pm 4.1$ & 0.71 \\
\hline Diabetes (n/\%) & $27(55.10)$ & $22(42.30)$ & 0.025 & $19(42.22)$ & $17(45.94)$ & 0.71 & $9(28.13)$ & $10(28.57)$ & 0.51 \\
\hline Hypertension (n/\%) & $29(59.18)$ & $28(53.84)$ & 0.040 & $19(41.30)$ & $20(54.05)$ & 0.013 & $14(43.75)$ & $12(34.28)$ & 0.011 \\
\hline Previous stroke $(\mathrm{n} / \%)$ & $19(38.77)$ & $14(26.92)$ & 0.010 & $16(34.78)$ & $10(27.02)$ & 0.041 & $11(34.37)$ & $9(25.71)$ & 0.027 \\
\hline Alx (\%) & 116. 3.5 & $112 \pm 2.8$ & 0.030 & $111 \pm 3.7$ & $108 \pm 2.9$ & 0.031 & $108 \pm 4.4$ & $107 \pm 3.5$ & 0.021 \\
\hline PWV (m/sec) & $14.45 \pm 2.6$ & $12.81 \pm 2.1$ & 0.030 & $11.2 \pm 2.31$ & $10.4 \pm 2.22$ & 0.030 & $11.48 \pm 1.98$ & $11.25 \pm 2,65$ & 0.030 \\
\hline NIHSS & $17.65 \pm 14.59$ & $15.64 \pm 8.73$ & 0.021 & $22.51 \pm 16.06$ & $19.00 \pm 12.72$ & 0.034 & $25.23 \pm 8.78$ & $22.23 \pm 8.78$ & 0.023 \\
\hline CAD (n/\%) & $18(36.73)$ & $14(26.92)$ & 0.011 & $16(35.5)$ & $12(32.43)$ & 0.71 & $8(25)$ & $8(20)$ & 0.07 \\
\hline $\begin{array}{l}\text { Micro-albuminuria } \\
\text { (n/\%) }\end{array}$ & $21(42.85)$ & $17(32.76)$ & 0.02 & $17(37.77)$ & $9(24.37)$ & 0.03 & $9(28.12)$ & $7(20)$ & 0.045 \\
\hline Carotid plaque (n/\%) & $14(28.57)$ & $9(17.30)$ & 0.031 & $16(35.55)$ & $11(29.79)$ & 0.023 & $7(21.87)$ & $5(14.28)$ & 0.02 \\
\hline LVH (n/\%) & $11(22.44)$ & $14(26.92)$ & 0.021 & $9(20)$ & $6(16.27)$ & 0.011 & $10(31.25)$ & $9(25.71)$ & 0.03 \\
\hline $\begin{array}{l}\text { Previous brain infarct } \\
\text { at neuro-imaging }\end{array}$ & $18(36.73)$ & $12(23.07)$ & 0.011 & $14(30.43)$ & $9(24.32)$ & 0.021 & $9(28.12)$ & $7(20)$ & 0.02 \\
\hline WMHLS (n/\%) & $19(38.77)$ & $14(26.92)$ & 0.021 & $13(28.26)$ & $8(21.62)$ & 0.032 & $11(34.37)$ & $8(22.85)$ & 0.021 \\
\hline CRP (mg/dl) & $4.1 \pm 2,3$ & $3.5 \pm 2.0$ & 0.011 & $5.0 \pm 1.8$ & $3.9 \pm 2.1$ & 0.018 & $5.8 \pm 1.6$ & $4.1 \pm 1.5$ & 0.020 \\
\hline IL-1-beta (pg/ml) & $7(5-11)$ & $5.2(3-8)$ & 0.012 & $9(5-12)$ & $7.9(4.5-12)$ & 0.017 & $12(6-17)$ & $10.8(6-14)$ & 0.021 \\
\hline IL-6 (pg/ml) & $9.8(6,5-19)$ & $8(6-21)$ & 0.021 & $12(6-27)$ & $10.4(5-23.5)$ & 0.014 & $13.5(5-31)$ & $10.2(4-26)$ & 0.019 \\
\hline TNF-a (pg/ml) & $29.5(9.5-33)$ & $23.5(8.5-29)$ & 0.025 & $41.5(11.25-47)$ & $37.5(11.6-41)$ & 0.031 & $43.5(21-58)$ & $35.5(20-50)$ & $<0.05$ \\
\hline E-selectin (ng/ml) & $5.05(1.8-5.7)$ & $3.25(1.7-5.7)$ & $<0.05$ & $4.85(1.96-7.9)$ & $2.75(1.6-5.6)$ & $<0.05$ & $4.15(2.2-4.1)$ & $3.55(2.1-4.1)$ & 0.011 \\
\hline P-selectin (ng/ml) & $4.5(2-6.8)$ & $3.1 \quad(2-4.7)$ & 0.022 & $5.4(2-8.0)$ & $4.3(2.7-6)$ & 0.034 & $6.2(3-8.9)$ & $4.9(2-7.5)$ & 0.018 \\
\hline VICAM-1 (ng/ml) & $19(10.1-21)$ & $15(8.1-22)$ & 0.031 & $17.5(9.2-20)$ & 15 (8.2-19.8) & 0.022 & $19(10.1-25)$ & $17.8(11.1-23)$ & 0.041 \\
\hline ICAM-1 (ng/ml) & $20.8(15.2-27)$ & $15.8(10.2-17)$ & $<0.05$ & $18.9(11.2-23)$ & $16.9(9.8-20)$ & 0.028 & $22.6(12.2-29)$ & $21.3(10-27)$ & 0.021 \\
\hline IL-10 (pg/ml) & $2.95(2-7)$ & $3.15(2-8)$ & 0.45 & $2.96(2-7)$ & $3.17(2-6)$ & 0.52 & $3.2(2-9)$ & $23.2(2-9)$ & 0.39 \\
\hline vWF (ng/ml) & $12(4-19)$ & $9(4-16)$ & $<0.05$ & $14(5-19)$ & $11(5-16)$ & $<0.05$ & $14(6-18)$ & $12(6-18)$ & 0.023 \\
\hline PAl-1 (pg/ml) & $145(97.5-178)$ & $137(98.5-149)$ & $<0.05$ & $139(79.4-165)$ & $128(91.5-135)$ & $<0.05$ & 139 (99.0-145) & 109 (99.0-145) & $<0.05$ \\
\hline TPA (pg/ml) & $28(16.15-39)$ & $24(12.15-35)$ & 0.021 & $26.7(11.4-31)$ & $22(10.5-29)$ & 0.031 & $24(12.1-47)$ & $20(10.1-39)$ & 0.021 \\
\hline
\end{tabular}

SBP: systolic blood pressure; DBP: diastolic blood pressure; MAP: mean arterial pressure; Alx: augmentation index; PWV: pulse wave velocity; SSS: Scandinavian Stroke Scale score; NIHSS: National Institutes of Health Stroke Scale ; Angiotensin II receptor blockers (ARBs); CAD: coronary artery disease; CHF: congestive heart failure; LVH: left ventricular hypertrophy; WMHLS: white matter hypertension lesions.

Demographic and history data are expressed as $n^{\circ}$ (percentage). 
(7-22) and lower median plasma values of IL-10 [ 11 $(6-15) \mathrm{pg} / \mathrm{ml})$ vs. 4 (3-9) $\mathrm{pg} / \mathrm{ml}$ vs. $2(1-5) \mathrm{pg} / \mathrm{ml} ; \mathrm{p}<$ 0.001] ( see Table 1).

Among subjects with acute ischemic stroke we observed higher plasma values of CRP, IL-1 $\beta$, IL-6, TNF- $\alpha$, E-Selectin, P-Selectin, ICAM-1, VCAM-1, wWF, PAI-1 in subjects with LAAS, lacunar and CEI subtype ischemic stroke and metabolic syndrome compared to subjects with this subtype of stroke without metabolic syndrome (see Table 2).

\section{Relationship between PWV and immune-inflammatory variables}

In subjects with acute ischemic stroke and metabolic syndrome compared to subjects with acute ischemic stroke without metabolic syndrome we observed a more significant positive correlation for age, and gender, between PWV and CRP ( $\mathrm{r}=0.38$ vs. $\mathrm{r}=0.31 ; \mathrm{p}=0.021)$; TNF- $\alpha(r=0.44$ vs. $\mathrm{r}=0.34 p<0.05), \operatorname{IL1} \beta(r=0.39$ vs. $\mathrm{r}=$ $0.30 ; p<<0.05)$, IL-6 ( $r=0.42$ vs. $\mathrm{r}=0.35 p=0.032)$, Eselectin $(r=0.36$ vs. $\mathrm{r}=0.29 ; p=0.038)$, P-selectin $(r=0.34$ vs. $\mathrm{r}=0.28 ; p=0.041)$, and IL-6 $(r=0.21 ; P<0.05)$, vWF $(r=0.40$ vs. $\mathrm{r}=0.34 ; p=0.019)$, PAI- 1 ( $\mathrm{r}=0.37$ vs. $\mathrm{r}=0.29$; $p<0.05$ ) (see Table 3).

\section{Relationship between Alx and and immune-inflammatory} variables

In subjects with acute ischemic stroke and metabolic syndrome compared to subjects with acute ischemic stroke without metabolic syndrome we observed a more significant positive correlation for age, and gender, between AIX and IL-6 ( $\mathrm{r}=0.33$ vs. $\mathrm{r}=0.23$; $\mathrm{p}=0.022$ ), TNF- $\alpha(r=0.32$ vs. $r=0.26 ; p=0.033)$ and vWF $(r=0.36$ vs. $\mathrm{r}=0.31 ; \mathrm{p}=0.031)$ (see Table 3 ).

\section{Relationship Between PWV and immune-inflammatory variables in relation to TOAST subtype}

Among subjects with lacunar subtype and metabolic syndrome, PWV (after correction for age and gender) was more significantly and positively related to CRP, IL$1 \beta$, IL-6, TNF- $\alpha$, vWF and PAI-1 (see Table 4) compared to subjects without metabolic syndrome and the same subtype of stroke.

In subjects with stroke classified as LAAS and metabolic syndrome, PWV was more significantly and positively related to CRP, IL- $1 \beta$, IL-6, TNF- $\alpha$, vWF and PAI1 (see Table 4) compared to subjects without metabolic syndrome and the same subtype of stroke.

In subjects with CEI subtype and metabolic syndrome, PWV was more significantly and positively related to vWF but not with TNF- $\alpha$, CRP, IL-6, IL-1 $\beta$ and PAI-1 (see Table 4) compared to subjects without metabolic syndrome and the same subtype of stroke.

Relationship between Alx and immune-inflammatory variables in relation to TOAST subtype

Among subjects with Lacunar subtype, AIx was more positively and significantly correlated (after correction for

Table 3 Correlations of PWv and Aix with immune-inflammatory variables

\begin{tabular}{|c|c|c|c|c|c|c|}
\hline \multirow{3}{*}{$\begin{array}{l}\text { Variable } \\
\text { Number }\end{array}$} & \multicolumn{2}{|c|}{ Pulse wave velocity } & \multirow{3}{*}{$p$} & \multicolumn{2}{|c|}{ Augmentation Index } & \multirow{3}{*}{$p$} \\
\hline & $\begin{array}{l}\text { With metabolic } \\
\text { syndrome }\end{array}$ & $\begin{array}{c}\text { Without metabolic } \\
\text { syndrome }\end{array}$ & & $\begin{array}{l}\text { With metabolic } \\
\text { syndrome }\end{array}$ & $\begin{array}{c}\text { Without metabolic } \\
\text { syndrome }\end{array}$ & \\
\hline & (n: 49) & (n: 52) & & (n: 45) & (n: 37) & \\
\hline CRP & 0.38 & 0.31 & 0.021 & 0.27 & 0.26 & 0.73 \\
\hline IL-1- $\beta$ & 0.39 & 0.30 & $<0.05$ & 0.21 & 0.23 & 0.66 \\
\hline IL-6 & 0.42 & 0.35 & 0.032 & 0.33 & 0.23 & 0.022 \\
\hline TNF- $a$ & 0.44 & 0.34 & $<0.05$ & 0.32 & 0.26 & 0.033 \\
\hline E-selectin & 0.36 & 0.29 & 0.038 & 0.19 & 0.21 & 0.56 \\
\hline P-selectin & 0.34 & 0.28 & 0.041 & 0.18 & 0.16 & 0.44 \\
\hline VICAM-1 & 0.30 & 0.29 & 0.70 & 0.20 & 0.22 & 0.66 \\
\hline ICAM-1 & 0.30 & 0.29 & 0.81 & 0.22 & 0.24 & 0.63 \\
\hline $\mathrm{IL}-10$ & 0.19 & 0.18 & 0.42 & 0.22 & 0.23 & 0.56 \\
\hline vWF & 0.40 & 0.34 & 0.019 & 0.36 & 0.31 & 0.031 \\
\hline PAI-1 & 0.37 & 0.29 & $<0.05$ & 0.18 & 0.15 & 0.77 \\
\hline TPA & 0.33 & 0.25 & 0.022 & 0.20 & 0.21 & 0.69 \\
\hline
\end{tabular}

Coefficients $(r)$ and $p$-values are calculated by the Pearson correlation mode.

SBP: systolic blood pressure ; DBP: diastolic blood pressure; MAP: mean arterial pressure; Alx: augmentation index; PWV: pulse wave velocity; SSS: Scandinavian Stroke Scale score; NIHSS: National Institutes of Health Stroke Scale; Angiotensin II receptor blockers (ARBs); CAD: coronary artery disease ; CHF: congestive heart failure; LVH: left ventricular hypertrophy; WMHLS: white matter hypertension lesions; TIA: transitory ischemic attack; SSS: Scandinavian Stroke Scale score; NIHSS: National Institutes of Health Stroke Scale; 
Table 4 Correlations of PWv with clinical variables and cardiovascular risk factors in relation to TOAST stroke subtype

\begin{tabular}{|c|c|c|c|c|c|c|c|c|c|}
\hline \multirow{3}{*}{ Variable } & \multicolumn{2}{|c|}{ Lacunar } & \multirow[b]{3}{*}{$p$} & \multicolumn{2}{|c|}{ LAAS } & \multirow[b]{3}{*}{$p$} & \multicolumn{2}{|c|}{ CEI } & \multirow[b]{3}{*}{$p$} \\
\hline & \multicolumn{2}{|c|}{ Pulse wave velocity (PWv) } & & \multicolumn{2}{|c|}{ Pulse wave velocity (PWv) } & & \multicolumn{2}{|c|}{ Pulse wave velocity (PWv) } & \\
\hline & $\begin{array}{l}\text { With metabolic } \\
\text { syndrome (n: } 49)\end{array}$ & $\begin{array}{c}\text { Without } \\
\text { metabolic } \\
\text { syndrome (n 52) }\end{array}$ & & $\begin{array}{l}\text { With metabolic } \\
\text { syndrome } \\
\text { (n: 45) }\end{array}$ & $\begin{array}{c}\text { Without } \\
\text { metabolic } \\
\text { syndrome (n: } 37)\end{array}$ & & $\begin{array}{l}\text { With metabolic } \\
\text { syndrome (n:32) }\end{array}$ & $\begin{array}{c}\text { Without } \\
\text { metabolic } \\
\text { syndrome }(n: 35)\end{array}$ & \\
\hline CRP & 0.32 & 0.27 & 0.031 & 0.38 & 0.32 & 0.041 & 0.29 & 0.28 & 0.63 \\
\hline IL-1- $\beta$ & 0.34 & 0.29 & 0.029 & 0.37 & 0.29 & $<0.05$ & 0.31 & 0.28 & 0.75 \\
\hline IL-6 & 0.33 & 0.28 & 0.030 & 0.35 & 0.32 & 0.041 & 0.29 & 0.28 & 0.63 \\
\hline TNF-a & 0.32 & 0.27 & 0.028 & 0.31 & 0.29 & $<0.05$ & 0.31 & 0.28 & 0.75 \\
\hline E-selectin & 0.32 & 0.23 & 0.65 & 0.31 & 0.23 & 0.041 & 0.19 & 0.20 & 0.61 \\
\hline P-selectin & 0.34 & 0.27 & 0.071 & 0.29 & 0.24 & $<0.05$ & 0.17 & 0.21 & 0.37 \\
\hline VICAM-1 & 0.33 & 0.29 & 0.060 & 0.36 & 0.26 & 0.041 & 0.19 & 0.20 & 0.61 \\
\hline ICAM-1 & 0.34 & 0.26 & 0.072 & 0.38 & 0.33 & 0.041 & 0.17 & 0.21 & 0.37 \\
\hline IL-10 & 0.20 & 0.22 & 0.56 & 0.21 & 0.23 & 0.66 & 0.23 & 0.25 & 0.57 \\
\hline vWF & 0.37 & 0.31 & 0.023 & 0.41 & 0.34 & $<0.05$ & 0.40 & 0.31 & $<0.05$ \\
\hline PAl-1 & 0.31 & 0.22 & 0.043 & 0.34 & 0.23 & 0.041 & 0.19 & 0.20 & 0.61 \\
\hline TPA & 0.29 & 0.26 & 0.55 & 0.26 & 0.24 & 0.75 & 0.20 & 0.21 & 0.37 \\
\hline
\end{tabular}

Coefficients $(r)$ and $p$-values are calculated by the Pearson correlation mode.

SBP: systolic blood pressure; DBP: diastolic blood pressure; MAP: mean arterial pressure; Alx: augmentation index; PWV: pulse wave velocity; SSS: Scandinavian Stroke Scale score; NIHSS: National Institutes of Health Stroke Scale; Angiotensin II receptor blockers (ARBs); CAD: coronary artery disease; CHF: congestive heart failure; LVH: left ventricular hypertrophy; WMHLS: white matter hypertension lesions.

Demographic and history data are expressed as $n^{\circ}$ (percentage).

Significant $R$ and $p$ are in bold.

age and gender) only to TNF- $\alpha$, IL- $1 \beta$ and vWF compared to subjects without metabolic syndrome; in subjects with LAAS subtype and metabolic syndrome we observed a more significant and positive relationship between AIx and IL-6. vWF and TNF- $\alpha$ compared to subjects without metabolic syndrome; in CEI group with metabolic syndrome IL- 6 , TNF- $\alpha$ and $v-$ WF were more positively and significantly related to AIx compared to subjects without metabolic syndrome (see Table 5).

\section{Discussion}

Our main findings were that patients with acute ischemic stroke and metabolic syndrome had both increased inflammatory markers and arterial stiffness indexes, compared to control subjects with acute ischemic stroke without metabolic syndrome.

This is the first study, to the best of our knowledge, to show in patients with acute ischemic stroke and metabolic syndrome that circulating levels of some immuneinflammatory markers such as CRP, IL-6, IL- $\beta$, TNF- $\alpha$ and VWF are more significantly correlated to PWV and wave reflection compared to subjects without metabolic syndrome.

The existence of a strong association between the presence of MetS and arterial stiffness has been shown in many cross-sectional studies $[20,21]$.

Inflammation is an important component of metabolic syndrome (MetS) which could be the link between the metabolic and cardiovascular consequences of this condition. Low-grade systemic inflammation, elevated leptin concentration and low adiponectin level are described in very young obese children, correlating with a range of variables of metabolic syndrome. Inflammation and adipocytokines can play an important role in the etiology of metabolic syndrome [21].

Results from some studies $[20,21]$ show that adiponectin concentrations are reduced with increased adiposity, while others show that TNF- $\alpha$, IL-6, resistin and CRP concentrations may be elevated with increased adiposity. A possible link exist between decreased adiponectin with increased insulin resistance, while some evidence links increased TNF- $\alpha$ and resistin with increased insulin resistance Another study relate higher blood pressures to decreased adiponectin, increased TNF- $\alpha$, and CRP concentrations [22].

Our findings, reporting higher plasma values of immune-inflammatory variables in subjects with acute ischemic stroke and metabolic syndrome, and more significant correlations between these immune-inflammatory variables and arterial stiffness markers in subjects with metabolic syndrome, underlines the role of inflammation in the vascular complications of metabolic syndrome.

Our findings show that both aortic stiffness and wave reflection are more related to the degree of systemic inflammation in stroke subjects with metabolic syndrome, suggesting that circulating inflammation mediators such as 
Table 5 Correlations of Alx with clinical variables and cardiovascular risk factors in relation to TOAST stroke subtype

\begin{tabular}{|c|c|c|c|c|c|c|c|c|c|}
\hline \multirow{3}{*}{ Variable } & \multicolumn{2}{|c|}{ Lacunar } & \multirow{3}{*}{$\begin{array}{l}p \\
0\end{array}$} & \multirow{2}{*}{\multicolumn{2}{|c|}{$\begin{array}{c}\text { LAAS } \\
\text { Augmentation Index (Alx) }\end{array}$}} & \multirow{3}{*}{$p$} & \multirow{2}{*}{\multicolumn{2}{|c|}{$\begin{array}{l}\text { CEI } \\
\text { ation Index (AIx) }\end{array}$}} & \multirow{3}{*}{$p$} \\
\hline & \multicolumn{2}{|c|}{ Augmentation Index (Alx) } & & & & & & & \\
\hline & $\begin{array}{l}\text { With metabolic } \\
\text { syndrome } \\
\text { (n: 45) }\end{array}$ & $\begin{array}{l}\text { Without metabolic } \\
\text { syndrome } \\
\text { (n: } 37)\end{array}$ & & $\begin{array}{l}\text { With metabolic } \\
\text { syndrome (n: 45) }\end{array}$ & $\begin{array}{c}\text { Without } \\
\text { metabolic } \\
\text { syndrome (n: 37) }\end{array}$ & & $\begin{array}{l}\text { With metabolic } \\
\text { syndrome } \\
(\mathrm{n}: 32)\end{array}$ & $\begin{array}{c}\text { Without } \\
\text { metabolic } \\
\text { syndrome }(n: 35)\end{array}$ & \\
\hline CRP & 0.24 & 0.26 & 0.73 & 0.27 & 0.26 & 0.81 & 0.20 & 0.21 & 0.73 \\
\hline IL-1- $\beta$ & 0.21 & 0.22 & 0.60 & 0.24 & 0.25 & 0.66 & 0.20 & 0.22 & 0.56 \\
\hline IL-6 & 0.31 & 0.25 & 0.026 & 0.34 & 0.226 & 0.011 & 0.33 & 0.23 & 0.022 \\
\hline TNF- $a$ & 0.33 & 0.27 & 0.022 & 0.32 & 0.26 & 0.033 & 0.32 & 0.26 & 0.033 \\
\hline $\begin{array}{l}\text { E- } \\
\text { selectin }\end{array}$ & 0.20 & 0.27 & 0.56 & 0.19 & 0.21 & 0.56 & 0.19 & 0.21 & 0.56 \\
\hline $\begin{array}{l}\mathrm{P} \text { - } \\
\text { selectin }\end{array}$ & 0.19 & 0.15 & 0.33 & 0.18 & 0.16 & 0.44 & 0.18 & 0.16 & 0.44 \\
\hline VICAM-1 & 0.21 & 0.22 & 0.66 & 0.22 & 0.21 & 0.66 & 0.20 & 0.22 & 0.66 \\
\hline ICAM-1 & 0.22 & 0.24 & 0.63 & 0.20 & 0.24 & 0.63 & 0.22 & 0.24 & 0.63 \\
\hline IL-10 & 0.22 & 0.23 & 0.56 & 0.22 & 0.23 & 0.56 & 0.22 & 0.23 & 0.56 \\
\hline vWF & 0.36 & 0.30 & 0.020 & 0.33 & 0.27 & 0.029 & 0.36 & 0.32 & 0.018 \\
\hline PAl-1 & 0.19 & 0.14 & 0.64 & 0.16 & 0.14 & 0.77 & 0.18 & 0.15 & 0.77 \\
\hline TPA & 0.22 & 0.25 & 0.77 & 0.20 & 0.21 & 0.69 & 0.20 & 0.21 & 0.69 \\
\hline
\end{tabular}

Coefficients $(r)$ and $p$-values are calculated by the Pearson correlation mode.

SBP: systolic blood pressure; DBP: diastolic blood pressure; MAP: mean arterial pressure; Alx: augmentation index; PWV: pulse wave velocity; SSS: Scandinavian Stroke Scale score; NIHSS: National Institutes of Health Stroke Scale ; Angiotensin II receptor blockers (ARBs); CAD: coronary artery disease; CHF: congestive heart failure; LVH: left ventricular hypertrophy; WMHLS: white matter hypertension lesions. Demographic and history data are expressed as $\mathrm{n}^{\circ}$ (percentage). Significant $R$ and $p$ are in bold.

CRP and some pro-inflammatory cytokines can influence the stiffness of vessels distant to those involved in the disease process itself.

This finding also emphasizes the systemic nature of the inflammatory response both in the atherosclerosis process and after acute ischemic stroke, encompassing biochemical and hemodynamic parameters. Cytokine plasma levels in their turn also influence vascular vulnerability to the inflammatory response and decreased production of the endogenous vasodilator nitric oxide (NO) [23], and it has been demonstrated that inhibition of basal NO synthesis increases aortic AIx75 and velocity in vivo [23].

An immune-inflammatory cascade occurs after an acute ischemic stroke [22,24], so, on this basis, cytokine levels that we reported and measured $72 \mathrm{hr}$ from symptom onset, express an acute rise in these inflammatory markers. What is the relationship between acute high levels of cytokines and arterial stiffness indexes in stroke patients in relation to the presence of metabolic syndrome? Does metabolic syndrome trigger a higher degree of hyper-acute inflammatory state after ischemic stroke directly causing an increase of arterial stiffness? Or in stroke-prone patients with a chronic inflammatory activation due to their metabolic background (insulin resistance, adipocite dysfunction) exacerbated by acute stressor events such an acute cerebrovascular event, does inflammation represent the pathogenic basis of higher arterial stiffness indexes levels in patients with acute ischemic stroke and metabolic syndrome compared to stroke patients without metabolic syndrome?

It's difficult to answer, maybe the peculiar metabolic and inflammatory background of metabolic syndrome may explain the observed relationship between immuneinflammatory markers, arterial stiffness indexes and acute ischemic stroke in metabolic syndrome patients.

In diabetes and metabolic syndrome exists a complex interrelationship of various inflammatory variables with metabolic disorders and their effect on the cardiovascular system. A simplified explanation can be that inflammation increases insulin resistance, which in turn leads to obesity while perpetuating diabetes, high blood pressure, prothrombotic state and dyslipidaemia [25]. Some studies $[26,27]$ have produced data suggesting an interplay between hormones, cytokines and resistin. Insulin resistance represents a common metabolic abnormality leading to cardiovascular disease and cerebrovascular complications. As several cytokines are also produced by adipose tissue [28] it was postulated that an "adipo-vascular" axis [29] may contribute to the increased risk of cardiovascular 
events in patients with metabolic syndrome. In patients with metabolic syndrome this "adipo-vascular axis" expresses itself in lower plasma levels of adiponectin and higher plasma levels of IL-6 that could be linked to the development of arterial stiffness and to the pathogenesis of ischemic stroke through microvascular and inflammatory mechanisms. Inflammation has been emphasized as a critical factor in the pathogenesis and destabilization of atherosclerotic lesions [30]. Early in the course of diabetes and metabolic syndrome intracellular hyperglycemia due to insulin resistance causes changes in blood flow and increased vascular permeability. This reflects decreased activity of nitric oxide, and increased activity of angiotensin II and endothelin-1 [31,32]. Angiotensin II produces acute vasoconstriction, leading to an increase in blood pressure. Endothelin-1 is also an important regulator of vascular tone and has been implicated in the pathogenesis of atherosclerosis. In addition, abnormalities of extracellular matrix contribute to an irreversible increase in vascular permeability. Hyperglycemia itself leads not only to decreased endothelial production of nitric oxide, which represents an anti-atherogenic molecule, but also to increased production of a potent inhibitor of fibrinolysis, namely plasminogen activator inhibitor 1 (PAI-1) [33].

Circulating inflammatory markers, such as hs-CRP, are known to be associated with cardiovascular disease and are useful for risk stratification of patients with cardiovascular disease $[34,35]$. However, circulating inflammatory markers cannot provide information on the vascular inflammation of local individual vascular lesions.

In subjects with acute ischemic stroke and metabolic syndrome we observed higher plasma values of immuneinflammatory markers in subjects with LAAS, lacunar and CEI subtype of ischemic stroke and metabolic syndrome compared to subjects with these subtypes of stroke without metabolic syndrome. This finding appears especially interesting in relation to the previous findings by our group [36-40] showing a higher degree of immune-inflammatory activation of the acute phase of stroke only in CEI subtype of stroke, whereas in patients with acute ischemic stroke and metabolic syndrome these three subtypes of stroke are characterized by a higher degree of immune-inflammatory activation compared to patients with acute ischemic stroke and without metabolic syndrome. It is as if metabolic background could trigger a high inflammatory cascade irrespective of stroke subtype and of its pathogenesis.

Nevertheless, it's possible to obtain an answer to the question about the relationship between inflammation markers and arterial stiffness indexes in acute ischemic stroke in subjects with metabolic syndrome by evaluating our findings of some significant correlations at intra-group analysis in each TOAST subtype between arterial stiffness indexes and immune-inflammatory markers. We observed a higher correlation between immune- inflammatory variables and arterial stiffness indexes in subjects with acute ischemic stroke and metabolic syndrome, whereas a subtype oriented analysis showed a higher relationship between inflammatory variables and PWV in LAAS, lacunar and CEI subtypes of stroke. In a previous study [36] by our group cardioembolic stroke appears as the "less atherosclerotic" among each diagnostic subtype of stroke, reporting the correlation of PWV only with CRP and vWF. In our subjects with acute ischemic stroke and metabolic syndrome, metabolic background and inflammatory pathways of these types of patients trigger a more intense immune-inflammatory activation irrespective of stroke subtype and this finding could appear original owing to the fact that no study to our knowledge evaluated inflammatory degree in patients with metabolic syndrome and an acute ischemic event such as ischemic stroke.

In conclusion, the results of our study support the view that patients with metabolic syndrome and acute ischemic stroke have a higher degree of immune-inflammatory markers strictly related to values of arterial stiffness indexes compared to stroke controls without metabolic syndrome and independently of stroke subtypes.

\section{Abbreviations}

SBP: Systolic blood pressure; DBP: Diastolic blood pressure; MAP: Mean arterial pressure; Alx: Augmentation index; PWV: Pulse wave velocity; SSS: Scandinavian Stroke Scale score; NIHSS: National Institutes of Health Stroke Scale; ARBs: Angiotensin II receptor blockers; CAD: Coronary artery disease; CHF: Congestive heart failure; TIA: Transitory ischemic attack; LVH: Left ventricular hypertrophy; WMHLS: White matter hypertensity lesions; VICAM-1: Vascular cell adhesion protein 1; ICAM-1: Intercellular Adhesion Molecule 1; IL-6: Interleukin-6; IL-1 beta: Interleukin 1 beta; IL-10: Interleukin-10; vWF: Von Willebrand Factor; PAl-1: Plasminogen Activator Inhibitor 1; TPA: Tissue plasminogen activator.

\section{Competing interests}

We have no conflict of interest or finantial disclosure.

\section{Authors' contributions}

Study design AT, RP, GL, AP. Acquisition of data: AT, RP, BC, RD, VA, CB, VD, $\mathrm{CM}$. Analysis and interpretation of data: AT, DD, RP, GL, AP. Manuscript preparation: AT, RP. All authors read and approved the final manuscript.

\section{Author details}

'Dipartimento Biomedico di Medicina Interna e Specialistica, Università degli Studi di Palermo, P.zza delle Cliniche n.2, 90127 Palermo, Italy. ${ }^{2}$ Dipartimento di Biomedicina Sperimentale e Neuroscienze Cliniche, Università degli Studi di Palermo, Palermo, Italy.

Received: 29 January 2014 Accepted: 18 February 2014 Published: 27 February 2014

\section{References}

1. Vaitkevicius PV, Fleg JL, Engel JH, O'Connor FC, Wright JG, Lakatta LE, et al: Effects of age and aerobic capacity on arterial stiffness in healthy adults. Circulation 1993, 88:1456-1462.

2. Boutouyrie P, Tropeano Al, Asmar R, Gautier I, Benetos A, Lacolley P, et al: Aortic stiffness is an independent predictor of primary coronary events in hypertensive patients: a longitudinal study. Hypertension 2002, 39:10-15.

3. Arnett DK, Boland LL, Evans GW, Riley W, Barnes R, Tyroler HA, et al: Hypertension and arterial stiffness: the Atherosclerosis Risk in Communities Study. ARIC Investigators. Am J Hypertens 2000, 13:317-323. 
4. Hingorani AD, Cross J, Kharbanda RK, Mullen MJ, Bhagat K, Taylor M, et al: Acute systemic inflammation impairs endothelium-dependent dilatation in humans. Circulation 2000, 102:994-999.

5. Bacon PA, Raza K, Banks MJ, Townend J, Kitas GD: The role of endothelial cell dysfunction in the cardiovascular mortality of RA. Int Rev Immunol 2002, 21:1-17.

6. Haverkate F, Thompson SG, Pyke SD, Gallimore JR, Pepys MB: Production of C-reactive protein and risk of coronary events in stable and unstable angina. European Concerted Action on Thrombosis and Disabilities Angina Pectoris Study Group. Lancet 1997, 349:462-466.

7. Lindahl B, Toss $H$, Siegbahn A, Venge $P$, Wallentin L: Markers of myocardial damage and inflammation in relation to long-term mortality in unstable coronary artery disease: FRISC Study Group. Fragmin during Instability in Coronary Artery Disease. N Engl J Med 2000, 343:1139-1147.

8. Kampus R, Kals J, Ristimãe T, Fischer K, Zilmer M, Teesalu R: High sensitivity $\mathrm{C}$-reactive protein affects central hemodynamics and augmentation index in apparently healthy persons. J Hypertens 2005, 22:1133-1139.

9. Booth AD, Wallace S, McEniery CM, Yasmin, Brown J, Jayne DR, Wilkinson IB: Inflammation and arterial stiffness in systemic vasculitis. A model of vascular inflammation. Arthritis Rheum 2004, 50:581-588.

10. Yasmin, McEniery CM, Wallace S, Mackenzie IS, Cockcroft JR, Wilkinson IB: C-reactive protein is associated with arterial stiffness in apparently healthy individuals. Arterioscler Thromb Vasc Biol 2004, 24:969-974.

11. Expert Panel on Detection, Evaluation, and Treatment of High Blood Cholesterol in Adults: Executive Summary of The Third Report of The National Cholesterol Education Program (NCEP) Expert Panel on Detection, Evaluation, And Treatment of High Blood Cholesterol In Adults (Adult Treatment Panel III). JAMA 2001, 285(19):2486-2497.

12. Satoh H, Kishi R, Tsutsui H: Metabolic syndrome is a significant and independent risk factor for increased arterial stiffness in Japanese subjects. Hypertens Res 2009, 32(12):1067-1071.

13. Bots ML, Dijk JM, Oren A, Grobbee DE: Carotid intima-media thickness, arterial stiffness and risk of cardiovascular disease: current evidence. $J$ Hypertens 2002, 20(12):2317-25.

14. Ahluwalia N, Drouet L, Ruidavets JB, Perret B, Amar J, Boccalon $H$, Hanaire- Broutin $\mathrm{H}$, Ferrieres J: Metabolic syndrome is associated with markers of subclinical atherosclerosis in a French population-based sample. Atherosclerosis 2006, 186(2):345-353.

15. Tuttolomondo A, Di Raimondo D, Di Sciacca R, Pecoraro R, Arnao V, Buttà C, Licata G, Pinto A: Arterial stiffness and ischemic stroke in subjects with and without metabolic syndrome. Atherosclerosis 2012, 225(1):216-219.

16. Teramura M, Emoto M, Araki T, Yokoyama H, Motoyama K, Shinohara K, Mori K, Koyama H, Shoji T, Inaba M, et al: Clinical impact of metabolic syndrome by modified NCEP-ATPIII criteria on carotid atherosclerosis in Japanese adults. J Atheroscler Thromb 2007, 14(4):172-178.

17. Hatano S: Experience from a multicenter Stroke register; a preliminary report. Bull World Health Organ 1976, 54:541e53.

18. Adams HP, Bendixen BH, Kappelle J, Biller J, Love B, Gordon MD, Marsh EE, and the TOAST Investigators: Classification of subtype of acute ischemic stroke. Stroke 1993, e24:358.

19. Kovaite M, Petrulioniene Z, Ryliskyte L, Badariene J, Dzenkeviciute V, Cypiene A, Laucevicius A, Polena S, Gintautas J: Systemic assessment of arterial wall structure and function in metabolic syndrome. Proc West Pharmacol Soc 2007, 50:123-130.

20. Teramura M, Emoto M, Araki T, Yokoyama H, Motoyama K, Shinohara K, Mori K, Koyama H, Shoji T, Inaba M, et al: Clinical impact of metabolic syndrome by modified NCEP-ATPIII criteria on carotid atherosclerosis in Japanese adults. J Atheroscler Thromb 2007, 14(4):172-178.

21. Bahia L, Aguiar LG, Villela N, Bottino D, Godoy-Matos AF, Geloneze B, Tambascia M, Bouskela E: Relationship between adipokines, inflammation, and vascular reactivity in lean controls and obese subjects with metabolic syndrome. Clinics (Sao Paulo) 2006, 61(5):433-40.

22. Ekdahl CT, Kokaia Z, Lindvall O: Brain inflammation and adult neurogenesis: the dual role of microglia. Neuroscience 2008, 158:1021-1029.

23. Valle M, Martos R, Gascón F, Cañete R, Zafra MA, Morales R: Low-grade systemic inflammation, hypoadiponectinemia and a high concentration of leptin are present in very young obese children, and correlate with metabolic syndrome. Diabetes Metab 2005, 31(1):55-62.

24. Welsh P, Lowe GD, Chalmers J, et al: Associations of proinflammatory cytokines with the risk of recurrent stroke. Stroke 2008, 39:2226-2230.
25. Bassuk SS, Rifai N, Ridker PM: High sensitivity C-reactive protein: clinical importance. Curr Probl Cardiol 2004, 29:439-493.

26. Wilkinson IB, MacCallum H, Cockcroft JR, Webb DJ: Inhibition of basal nitric oxide synthesis increases aortic augmentation index and pulse wave velocity in vivo. Br J Clin Pharmacol 2002, 53:189-192.

27. Sell H, Eckel J: Monocyte chemotactic protein-1 and its role in insulin resistance. Curr Opin Lipidol 2007, 18(3):258-62.

28. Dyck DJ, Heigenhauser GJ, Bruce CR: The role of adipokines as regulators of skeletal muscle fatty acid metabolism and insulin sensitivity. Acta Physiol (Oxf) 2006, 186(1):5-16

29. Heilbronn LK, Campbell LV: Adipose tissue macrophages, low grade inflammation and insulin resistance in human obesity. Curr Pharm Des 2008, 14(12):1225-1230.

30. Ross R: Atherosclerosis: an inflammatory disease. N Engl J Med 1999, 340:115-126.

31. Frühbeck G, Gomez-Ambrosi J, Muruzabal FJ, Burrell MA: The adipocyte:a model for integration of endocrine and metabolic signaling in energy metabolism regulation. Am J Physiol 2001, 280:E827-E847.

32. Matsuda M, Shimomura I, Sata M, Arita Y, Nishida M, Maeda N, Kumada M, Okamoto Y, Nagaretani H, Nishizawa H, Kishida K, Komuro R, Ouchi N, Kihara S, Nagai R, Funahashi T, Matsuzawa Y: Role of adiponectin in preventing vascular stenosis. J Biol Chem 2002, 277:37487-37491.

33. Brownlee M: Biochemistry and molecular cell biology of diabetic complications. Nature 2001, 414:813-820.

34. Ridker PM: C-reactive protein and the prediction of cardiovascular events among those at intermediate risk: moving an inflammatory hypothesis toward consensus. J Am Coll Cardiol 2007, 49:2129-2138.

35. Sell H: Eckel Chemotactic cytokines, obesity and type 2 diabetes: in vivo and in vitro evidence for a possible causal correlation? J Proc Nutr Soc 2009, 24:1-7.

36. Bastard JP, Maachi M, Lagathu C, Kim MJ, Caron M, Vidal H, Capeau J, Feve $B$ : Recent advances in the relationship between obesity, inflammation, and insulin resistance. Eur Cytokine Netw 2006, 17(1):4-12.

37. Licata G, Tuttolomondo A, Di Raimondo D, Corrao S, Di Sciacca R, Pinto A: Immuno-inflammatory activation in acute cardio-embolic strokes in comparison with other subtypes of ischaemic stroke. Thromb Haemost 2009, 101(5):929-937.

38. Tuttolomondo A, Di Sciacca R, Di Raimondo D, Serio A, D'Aguanno G, La Placa S, Pecoraro R, Arnao V, Marino L, Monaco S, Natalè E, Licata G, Pinto A: Plasma levels of inflammatory and thrombotic/fibrinolytic markers in acute ischemic strokes: relationship with TOAST subtype, outcome and infarct site. J Neuroimmunol 2009, 215(1-2):84-89.

39. Tuttolomondo A, Di Raimondo D, Forte Gl, Casuccio A, Vaccarino L, Scola L, Pecoraro R, Serio A, Clemente G, Arnao V, Palmeri M, Misiano G, Lio D, Pinto A, Licata G: Single nucleotide polymorphisms (SNPs) of pro-inflammatory/anti-inflammatory and thrombotic/fibrinolytic genes in patients with acute ischemic stroke in relation to TOAST subtype. Cytokine 2012, 58(3):398-405.

40. Davì G, Tuttolomondo A, Santilli F, Basili S, Ferrante E, Di Raimondo D, Pinto A: Licata GCD40 ligand and MCP-1 as predictors of cardiovascular events in diabetic patients with stroke. J Atheroscler Thromb 2009, 16(6):707-13.

\section{doi:10.1186/1758-5996-6-28}

Cite this article as: Tuttolomondo et al:: Immune-inflammatory markers and arterial stiffness indexes in subjects with acute ischemic stroke with and without metabolic syndrome. Diabetology \& Metabolic Syndrome 2014 6:28. 